\title{
INDEX OF NAMES OF SCHOLARS
}

Abercrombie, Nigel 513n

Abicht, Ludo 211n

Adorno, Theodor W. 431

Agamben, Giorgio 31, 362, 363

Aikin, John 173n

Akkerman, Fokke 521, 523

Albach, Ben 140n, 149n, 157n, 159n, 160n, 162n, 165n, 166n, 167n, 285, 286, 287n, 290, 293n, 298, 299, 309n, $312 \mathrm{n}, 313 \mathrm{n}, 500 \mathrm{n}, 501 \mathrm{n}, 502 \mathrm{n}, 504 \mathrm{n}$

Alberdingk Thijm, Jozef Albert 16, 145n, 165, 198

Allen, Don Cameron 509n, 510n

Allen, Graham 271n,

Alpers, Svetlana 465n

Alphen, Ernst van 21,37n

Alt, Peter-André $124 \mathrm{n}$

Amir, Ton 162n

Ankersmit, Frank R. 39, 40

Arendt, Hannah 38

Asperen, Bob van 150n

Asselbergs, Willem see Duinkerken, Anton van

Assmann, Aleida 125n

Assmann, Jan 331n

Astell, Ann W. 226n

Auerbach, Erich 268n, 385

Austin, John L. 128n

Bakker, Boudewijn 110n, 384n

Bal, Mieke 25, 29n, 32n, 42-43, 318, 319n, 320n, 322n, 324, 325, 330-31, $334,336 n, 337 n, 427 n, 432,438 n$, $478 \mathrm{n}$

Balkin, Jack M., 479, 480n

Barnouw, A.J. 101n

Barthes, Roland 271, 498n

Bataille, George 445

Baumgartner, Alexander 198

Bax, M.M.H. 390n, 391

Beardsworth, Richard 362

Beckerman, Bernard 303n

Beekman, Klaus 109n, 231n

Beer, Taco H. de 198n

Bekker, Hugo 380n

Belsey, Catherine 24, 26

Benjamin, Walter 26, 226, 227, 235, 327, $332,336,337,338,339,447,455,457$
Benoy, Peter 168n

Berg, Willem van den 23n, $164 n$

Bertram, Paul 501n

Bhabha, Homi K. 259n

Blakely, Allison $34 \mathrm{n}$

Bleeker, Maaike 67n, 322n, 323, 324n, $334 n, 335 n, 436,437$

Blijstra, Reinder 493

Bloemendal, Jan $71 \mathrm{n}, 74 \mathrm{n}, 82 \mathrm{n}, 117 \mathrm{n}$, 279n, 331n, 342n, 344n, 349n, 351n

Blok, Marja 113n

Bloom, Harold 272

Blumenberg, Hans 26n

Bolter, J. David 506n

Bomhoff, J.G. 18, 20, 233

Borch-Jacobson, Mikkel 357n

Bordoli, Roberto 523n

Bornemann, Ulrich 176n

Bostoen, Karel 120n

Bot, Jaap 109n

Böttiger, Carl Wilhelm 197n

Bouchard, Larry 30n

Bourdieu, Pierre 273

Bowring, John 173, 175, 469, 470

Braak, Menno ter 7, 8

Brachin, Pierre 326

Bradford, Richard 398

Brandt, George W. 107n, 108n

Brannigan, John 205

Brennan, Teresa 319

Brom, Gerard 17, 85n, 96n, 383n, 385n, 392n, 442n, 512n, 513n

Bruch, Hettel 493

Brugmans, H. 109n

Bruinsma, Henry A. $147 \mathrm{n}$

Bunge, Wiep van 82n, 514n, 523n

Burke, Peter 207n

Busken Huet, Conrad 208, 309

Butler, Judith 128n

Calis, Piet $86,89 n, 98 n, 101 n, 114 n$, 208n, 461n, 462, 463n, 512n

Cardozo, Benjamin N. 460n

Carlson, M. 286

Carre, Meyrick H. 427n

Cerquiglini, Bernard 496

Certeau, Michel de 25

Cohen, Barbara 25n 
Cohen, Stephen 234n

Cohen, Tom 25n

Cordes, Rudolf 149n

Cornelissen, J.D.M. 471, 472

Cover, Robert 475, 483n

Cowan, Bainard 336

Cox, Roger Lindsay 30n

Craik, Katherine 419, 420n

Culler, Jonathan 361n, 427n, 429n, 432

Davies, Gwendolyn 195n

Davis, Robert Con 261n

Davis, Tracey 323, 334

Deleuze, Gilles 26, 40, 359, 360n, 514n

Derrida, Jacques $269,359,361,362,363$, 375, 428n, 429, 457

Deursen, A.Th. van $87 n$, $91 n$

Deuss, Bart 289n

Dietz, Feike 411n

Diferee, Hendrik C. 173, 174, 198n, 493

Dijk, Hans van $142 n$

Dijk, Harry S. van 175n

Dijkhuizen, Jan Frans van 195n, 422n

Dixhoorn, Arjan van 103n, 342n

Dongelmans, Berry P.M. 492n, 493n

Drabbe, G.F. $85 \mathrm{n}$

Dubrow, Heather 234

Duinkerken, Anton van 15n, 364n, 366n, 372

Duits, Henk 154n, 233n, 377, 378n, 465n, 472, 473, 474, 478, 479

Dülmen, Richard van $132 \mathrm{n}$

Duyse, Florimond van 145n

Dyk, Harry S. van 175n, 470n

Eagleton, Terry $362,492 \mathrm{n}$

Eco, Umberto 273, 328

Edmundson, George 195n

Eisenstein, Elizabeth 497n

Elias, Johan E. 207n

Elias, W. 525n

Erenstein, Rob L. 149n

Eversmann, Peter G.F. 68n, 293n

Eyffinger, Arthur C. 478n, 485n

Fallon, Stephen M. 402

Ferguson, Robert A. 476

Ferris, David S. 26n

Filmer, Robert 389

Findlen, Paula 45

Fink, Josef 509n

Finke, Laurie 261n

Fischer-Lichte, Erika 115, 116n

Fish, Stanley 273, 395, 396n
Flemming, Willi 178n, 194n

Fletcher, Angus 227n

Foucault, Michel 27n, 271, 359, 363

Fredriksen, Paula 94n

Freud, Sigmund 319n, 433, 448, 449-52, 453, 456

Führer, Heidrun 196n

Gaakeer, Jeanne 233n, 460n, 499n

Gadamer, Hans-Georg 428n, 429

Garcia Martínez, Florentino 509n

Geerts, A.M.F.B. 34

Geertz, Clifford 479

Geesink, Marja S. 106n, 107n, 158n, 502n

Gelder, H.A. Enno van 207n, 208n

Gelderblom, Arie 126n, 359n

Gellrich, Jesse M. 385n

Gemert, Guillaume van 176n, 177n, 183n, 194n, 198n

Gemert, Lia (E.M.P.) van 11n, 20, 130n, 139n, 140n, 141n, 157n, 203n, 278n, 297n, 309n, 359n, 411n, 473, 474, 502

Gemmette, Elizabeth Villiers 460n

Genette, Gérard 272

Gerbrandy, Piet 410, 411n

Gerritsen, Johan 494n, 498n, 500n

Girard, René 132, 133

Goudriaan, Aza 512n

Graft, C. Catharina van de 342n

Graham, Joseph F. 254n

Grant, Michael 213, 219n

Green, André 449n

Greenblatt, Stephen 24, 115

Greg, W.W. 507n

Gregory, Brad 94n

Grijp, Louis Peter 139, 140n, 141n, $143 n, 144,145 n, 149 n, 152 n, 153 n$, $297 \mathrm{n}$

Groen, Marisa 162n

Grootes, Eddy K. x, 23, 113n, 117n, 118 n, 235n, 317n, 344n

Grüttemeier, Ralf 109n, 231n

Gullan-Whurr, Margaret 522n

Haas, Anna S. de 13n, 160n

Habbema, Cox 170n

Habermas, Jürgen 486

Haerten, Heinz 194n

Hasselbach, P.B. 301, 309

Haven, Korneel van der 159n, 501n

Hechtle, Martha 194n

Heidegger, Martin 428n, 441n

Heijer, Jac. 170n 
Hell, Maarten 109n

Hellinga, Wytze Gs. 112, 113n, 494n, 495, 498n

Helmers, Helmer J. 172n, 195n, 225n, 232n, 424n

Hermans, Theo ix, $1 \mathrm{n}$

Hoekveld-Meijer, Gerda 474

Hogendoorn, Wiebe 107n, 108n, 313n

Holly, Michael Ann 323

Hoogers, Gerard 441n

Hoppenbrouwers, F.J. 15

Horst, Carl 336

Hsia, R. Po-Chia 87n, 211

Hummelen, Wim M.H. 105, 107n, 292n

Hunt, Barbara Joan 30n

Huussen, Aarend H. 210n, 211n, $222,223 n$

Huyssen, Andreas 29n

IJsewijn, Jozef 349n, 350n

Ingen, Ferdinand van 178n, 181n, $183 n, 188 n$

Iser, Wolfgang $273,478 \mathrm{n}$

Israel, Jonathan 206n, 211, 228n, 525n

Jackson, Ken 379

Jakobson, Roman 252n

Jansen, Jeroen $255 n$

Jardine, Lisa 24

Jay, Martin 319n

Jauss, Hans Robert 45

Johannes, Wilhelm 183n

Johnson, Barbara 362, 375

Jonckbloet, W.J.A. 377n

Jones, Ernest 449

Junkers, Herbert 194n

Kalff, Gerrit 202, 494n, 499n

Kamps, Ivo 230n

Kant, Immanuel 375

Kantorowicz, Ernst H. 119n

Kaplan, Benjamin J. 99n

Kastan, David Scott 403n, 491, 492n, 506n

Kemperink, R.M. 231n, 232n, 499n

Kiedroń, Stefan 177n, 178n, 194n

King, John 395n

King, Peter $18,377 n, 379,389 n, 412 n$, 415n, 418

Kipka, Karl 184n, 345n, 347n

Kirkconnell, Watson 191n

Klausnitzer, Ralf 120n

Klerk, Cornelis R. de 206, 207, 208n, 209
Kliman, Bernice W. 501n

Knapen, Ben 228n, 229n

Knuvelder, Gerard 202

Koch, Elke 127n

Kock, Petra de 170n

Kok, A.S. 526

Kollewijn, Roeland A. 194n

Konst, Jan W.H. 20, 112n, 116n, 120n, $127 \mathrm{n}, 128 \mathrm{n}, 129 \mathrm{n}, 130 \mathrm{n}, 202 \mathrm{n}, 277 \mathrm{n}$, $377 n, 408,410,414 n, 419 n, 421,424 n$, 440, 495n, 525n, 526

Koppenol, Johan 225, 276n

Korsten, Frans-Willem 21, 22, 35n, 67n, $86,115 \mathrm{n}, 117 \mathrm{n}, 128 \mathrm{n}, 130 \mathrm{n}, 134 \mathrm{n}$, $179 n, 264 n, 277 n, 324 n, 356 n, 358 n$, 359n, 360, 366n, 369, 373, 375, 376, 378, 379, 385n, 390n, 391, 393, 409, $411 \mathrm{n}, 414,415 \mathrm{n}, 421,435,440 \mathrm{n}, 444 \mathrm{n}$, $447,458,465 \mathrm{n}, 474,475 \mathrm{n}, 477,484$, 494n, 495n, 504n, 515, 516, 521, 522n

Krispyn, Egbert 180n, 181n, 194n

Kristeva, Julia 30, 271

Kritzinger, M.S.B. 394n

Kuijpers, Erika 102n

Lachmann, Karl 496

Lacoue-Labarthe, Philippe 445, 452,456

Langvik-Johannessen, Kåre 18, 136n, 164n, 179n, 190n, 377n, 490n, 504n, $510 \mathrm{n}[+-]$

Leemans, E.A. $522 \mathrm{n}$

Leemans, Inger $525 \mathrm{n}$

Leendertz, P. 86n, 91n, 491n, 494n

Lennep, Jacob van 16-17, 161n, 162, 163n, 164, 201-02, 203, 377n, 490n, 492-93, 498n

Leuvensteijn, Arjan van 420n

Levinas, Emmanuel 375

Levinson, Marjorie 234n

Lieburg, Fred van $512 \mathrm{n}$

Littau, Karin 430n, 444

Loewenstein, David 395n, 397, 401

Lommel, A. van 89n, 96n

Louvat-Molozay, Bénédicte 143n

Luhmann, Niklas 460n

Lulofs, B.H. 15, 470

Luria, Keith $95 \mathrm{n}$

Lützelschwab, Ralf $127 \mathrm{n}$

Lyotard, Jean-François 449, 451, 452

Maljaars, Abraham 276n, 277n

Man, Paul De 25, 360n, 362, 431

Marcus, Leah S. 498n

Markell, Pratchen 38n 
Marnef, Guido $101 \mathrm{n}$

Marotti, Arthur 115n, 379

Martin, Henri-Jean 497n

Mathijsen, Marita 495n, 496n

McGann, Jerome 23n, 24

Méchoulan, Henry 207n, 208n

Meijer Drees, Marijke 133n, 225n, 229n, 232n, 233n, 360

Meijer, Fik 216n, 217n

Meijer, Maaike 210n, 211, 272

Meinsma, K.O. 522n

Melles, J. 86n

Meulenbroek, B.L. 489n

Michelfelder, Diane P. 429n

Michels, L.C. 17

Mignini, Filippo 522

Miller, J. Hillis 362

Mitchell, William J.T. 323, 361

Mody, Jehangir R.P. $195 n$

Molkenboer O.P., B.H. 13n, 17, 85n, 202n, 209, 491n, 492n, 496, 509n, 512n

Moller, H.W.E. 17

Mooij, Jan J.A. 479n

Moolhuizen, Jan Jurrien 195

Moréri, Louis 171, 172n

Morrison, Toni 34

Müller, August 195n

Müller Hofstede, Justus 319n

Nadler, Steven 212, 213n, 214, 216n, 515,522

Nellen Henk J.M. 98n, 211n

Nichols, Stephen G. 497

Nierop, Henk F.K. van $87 \mathrm{n}$

Noak, Bettina 116n, 119n, 124n, 125n, 128n, 134n, 184n, 377, 378n

Noë, Joris $377,383 n$

Noske, Frits R. 150n

Nyquist, Mary 489n

Oey-de Vita, Elise 106n, 107n, 158n 491n, 494n, 499n, 502n

Opsomer, Geert 168n

Orwell, George 23

Osterkamp, Ernst 379n, 390n

Otegem, Matthijs van $384 n$, $524 n$

Palmer, Richard E. 429n

Panofsky, Erwin 323

Parente, Jr., James A. 18, 71n, 275n, $341 n, 343 n, 348 n, 353 n, 355 n$, $377 n, 421 n$

Parry, Graham 395n
Peeters, Frank 168n

Pelt, Joke M. van $167 \mathrm{n}$

Pensky, Max 26n

Pether, Penelope J. 476n

Phillips, James E. 345n, 346n, 347n, 348n, 353n, 431n

Pieters, Jürgen 24, 26, 46, 55n, 115n, 205n, 359, 360n

Plard, Henri $178 \mathrm{n}$

Plas, Michel van der $198 \mathrm{n}$

Poelhekke, Jan J. 472n

Pollard, Tanya 420, 421n, 423

Pollmann, Judith S. 71n, 88n, 89n, 93n, 95n, 96n, 342n

Poole, William 380, 396n

Porteman, Karel 108n, 112n, 117n, 158n, 167n, 203, 344n, 461n, 463n, 465n, 504

Posner, Richard A. 429n

Postma, Hugo 113n

Pott, Clarence C. 194n

Potter, Lois 226n, 227n, 240

Poulssen, J. 20

Powell, John S. 143n

Prak, Maarten 102n

Prandoni, Marco 68n, 124n, 275n, 276n, 288, 309n, 312n, 314n

Pranger, M. Burcht 30n

Rademaker ss.cc., Cor S.M. 117n, $351 n$

Ramakers, Bart A.M. 142n

Rancière, Jacques 362, 363, 431, 432n

Rappaport, Herman 456n

Rasch, Rudolf A. 139n, 143n, 153n, 161n

Rasmussen, Mark David 234n

Raymond, Joad 395n

Rekers, Guus 21, 168, 170n

Rener, Frederick M. 252, 253n

Rens, Lieven 19, 194n, 471, 473

Riccoboni, Louis 312, 313n

Richards, I.A. 430

Roegiers, Jan 513n

Romein, Jan 86

Romein-Verschoor, Annie 86

Rooden, Peter van 211n

Rose, Mary Beth 413

Rowe, Katherine 410n

Rowen, Herbert H. 206n

Sabbe, Maurits 196n

Savile, Anthony 23n

Schaeffer, Jean-Mary 29, 226n, 432 
Schenkeveld-van der Dussen, Riet $\mathrm{x}$, 10n, 11n, 21n, 31n, 344n, 360, 375, 376n, 409, 490n, 491n, 494n, 495n, 510n, 516, 526

Schings, Hans-Jürgen 138n

Schleiermacher, Friedrich 253, 254

Schmitt, Carl 441n

Schoenfeldt, Michael 420n

Schöffer, Ivo 465n, 467n, 469n

Scholz-Heerspink, Myra 280n, 386n

Schößler, Franziska 115n, 133n

Schrant, J.M. 15

Schravendeel, Rogier $164 \mathrm{n}$

Schuytvlot, A.C. 10n, 490n, 492n, 496n, 500n, 501n, 502n, 504n, 505n

Scott, Joan Wallach 409-10

Sedgwick, Alexander 513n

Sellin, Paul R. 462n

Shapiro, James 222

Sharpe, K. 226n, 234n

Sierhuis, Freya 227n, 243n, 323n

Simoni, Anna E.C. 470n

Simons, L. 165, 422n

Skinner, Quentin 45

Smet, R. De 525n

Smit, Wisse A.P. passim

Smits-Veldt, Mieke B. x, 31n, 45n, 101n, $104 n, 106 n, 107 n, 109 n, 112 n, 117 n$, $157 \mathrm{n}, 158 \mathrm{n}, 160 \mathrm{n}, 162 \mathrm{n}, 163 \mathrm{n}, 203$, 232n, 235n, 236n, 240n, 274n, 276n, 278n, 292n, 293n, 299n, 312n, 344n, 394n, 461n, 463n, 465n, 495n, 500, 502n

Sneller, A. Agnes 408, 409, 410, 414, 494n, 495n

Spaans, Joke 87n, 96n

Spiertz, M.G. 513n

Spies, Marijke $x, 13 n, 18 n, 57 n, 60 n$, 76n, 85n, 101n, 104n, 108n, 109n, $117 \mathrm{n}, 235 \mathrm{n}, 325 \mathrm{n}, 359,360,463 \mathrm{n}$, $467 n, 482 n, 493,495 n$

Starobinski, Jean 449

Stein, Louise K. 143n

Steiner, George 428, 429n

Sterck, J.F.M. 17, 89n, 150n, 154n, 208, $209,502 n$

Sterne, Jill 377

Sternfeld, Frederick W. 139n

Stevens, Wallace 459n

Stipriaan, René van 226n, 245, 277n

Strengholt, Leendert 278n

Stronks, Els 170n

Szarota, Elida M. 277n

Szyrocki, Marian 178n
Tal, Kali 37n

Tambling, Jeremy 226n, 227n

Tanner, Marie 510n

Tate, Jr, Charles Delmer 489n

Tex, Jan den 228n, 229n, 238n

Thomas, Brook 476, 485

Thys, Walter 196n

Tilmans, Karin 469n

Todorov, Tzvetan 29n

Toulmin, Stephen 480n

Tracy, James D. 228n

Uit den Bogaard, Max Th. 474n

Unger, J.H.W. 232, 490n, 492n, 493n, 496n, 499n, 501n, 502n, 504n, 505n

Vaeck, Marc Van 494n

Valk, J. de 92n

Valk, Th. De 522n

Valkenburg, Jochem $167 \mathrm{n}$

Vandervelden, Jos 480, 481

Väth, Alfons 138n

Veldhorst, Natascha $139,140 \mathrm{n}, 141 \mathrm{n}$, $142 \mathrm{n}, 143 \mathrm{n}, 148 \mathrm{n}, 152 \mathrm{n}, 153$, $154 n, 297 n$

Venuti, Lawrence 254, 256, 264

Verheul, J.P. 483n

Verhofstadt, Edward 194n

Verwey, Albert 17, 206, 209, 265, 422n, 493

Visconsi, Elliott $476,477 \mathrm{n}$

Vloten, Johannes van 16, 493

Vollenhoven, C. van 250n

Vries, T. de 195n

Waal, Henri van de $111 \mathrm{n}, 112 \mathrm{n}$

Walch, Johannes L. 499n

Walcott, Derek 34

Wattel, Evert 420n

Weevers, Theodoor 194n, 445n

West, Martin L. 496n

Weststeijn, Thijs 110n

White, Hayden 39

Wigmore, John H. 460n

Wijnman, H.F. $97 \mathrm{n}$

Winkel, Jan W. te $202,377 \mathrm{n}$

Winnicot, David W. 433

Wiskerke, Evert Matthijs 13n

Witsen Geysbeek, Pieter G. 14, 15, 461n, 464n, 470, 486, 499n

Witstein, Sonja F. $117 \mathrm{n}$

Woerner, Roman 345n, 346, 347n, $353 n$

Wolfson, Susan $234 n$ 
Worden, Blair 395n, 477

Worp, Jacob A. 99n, 139n

Zalm, Rob van der 165n, 166n

Zijderveld, A. 522n
Zijlstra, S. 89n, 90n, 91n

Zilverberg, S.B.J. 522n

Žižek, Slavoj 433n

Zuckert, Catherine 428n

Zwicker, Steven N. 226, 234n 